\title{
Mesophase Control of Mesoporous Silica Thin Films by Vapor-phase Preparation
}

\author{
Shunsuke Tanaka*1,† and Hugh W. Hillhouse ${ }^{2}$ \\ ${ }^{1}$ Division of Chemical Engineering, Graduate School of Engineering Science, Osaka University, \\ 1-3 Machikaneyama, Toyonaka, Osaka 560-8531 \\ ${ }^{2}$ School of Chemical Engineering, Purdue University, \\ 480 Stadium Mall Drive, West Lafayette, Indiana 47907, U.S.A.
}

(Received May 25, 2006; CL-060611; E-mail: s060002@ipcku.kansai-u.ac.jp)

Highly ordered mesoporous silica thin films with $c 2 \mathrm{~mm}$ rectangular and $R 3 m$ rhombohedral symmetry have been synthesized on a silicon substrate by a vapor-phase method using $\mathrm{PEO}_{106}-\mathrm{PPO}_{70}-\mathrm{PEO}_{106}$ triblock copolymer as a templating agent.

Surfactant-templated mesoporous silica has attracted much attention because of their prospects for microelectronic low dielectric constant ${ }^{1}$ and photonic low refractive index ${ }^{2}$ materials. Ordered mesoporous silica films with various unique structures, including lamellar, 2D hexagonal $p 6 \mathrm{~mm}$ (thermal-induced to rectangular $c 2 m m),{ }^{3} 3 \mathrm{D}$ hexagonal $p 6_{3} / m m c,{ }^{3}$ bicontinuous cubic $I a 3 d,{ }^{4}$ primitive cubic Pm $3 n,{ }^{3}$ orthorhombic Fmmm, ${ }^{2}$ tetragonal $I 4 / \mathrm{mmm},{ }^{5}$ rhombohedral $R 3 m,{ }^{6,7}$ have been synthesized using the technique of evaporation-induced self assembly (EISA). ${ }^{8}$ Typically, the films are prepared by dip- or spin-coating with an acidic solution of a templating agent such as cetyltrimethylammonium bromide (CTAB) or block copolymer, a silica precursor such as tetraethyl orthosilicate (TEOS), and a water-miscible, volatile solvent (e.g., ethanol). Upon rapid solvent evaporation, at a certain concentration cooperative self-assembly of the surfactant/silicate leads to mesostructures.

On the contrary, the vapor-phase synthesis reported by Nishiyama and co-workers ${ }^{9}$ performed contacting the template films with hydrolyzed TEOS vapor at $90-120^{\circ} \mathrm{C}$ to prepare the mesostructured silica films. The TEOS molecules infiltrate into the surfactant film and the TEOS/surfactant composites dynamically rearrange, resulting in the highly ordered mesophase. However, no preparation procedure has been reported to control the mesostructure of the films using the same templating agent.

In the present study, we have applied the vapor-phase preparation to the control of mesophase topology and obtained the silica films with $c 2 m m$ and $R 3 m$ symmetry using $\mathrm{PEO}_{106^{-}}$ $\mathrm{PPO}_{70}-\mathrm{PEO}_{106}$ (Pluronic F127) copolymer. The phase topology, order, and orientation of the films have identified by the combination of grazing angle of incidence small-angle X-ray scattering (GISAXS) and high-resolution field emission scanning electron microscope (FESEM) measurements. The measured scattering patterns were simulated using NANOCELL, ${ }^{10}$ a Mathematicabased program using the distorted wave Born approximation. The predicted spot patterns from NANOCELL are directly overlaid on the GISAXS patterns. The detailed synthesis and characterization methods are shown in the references and notes section. $^{11}$

The two types of periodically organized mesoporous silica films (MSF) prepared were named accordingly with the TEOS infiltration temperature and time. Hence, the silica films prepared at $90^{\circ} \mathrm{C}$ for $1 \mathrm{~h}$ are labeled MSF90(1).
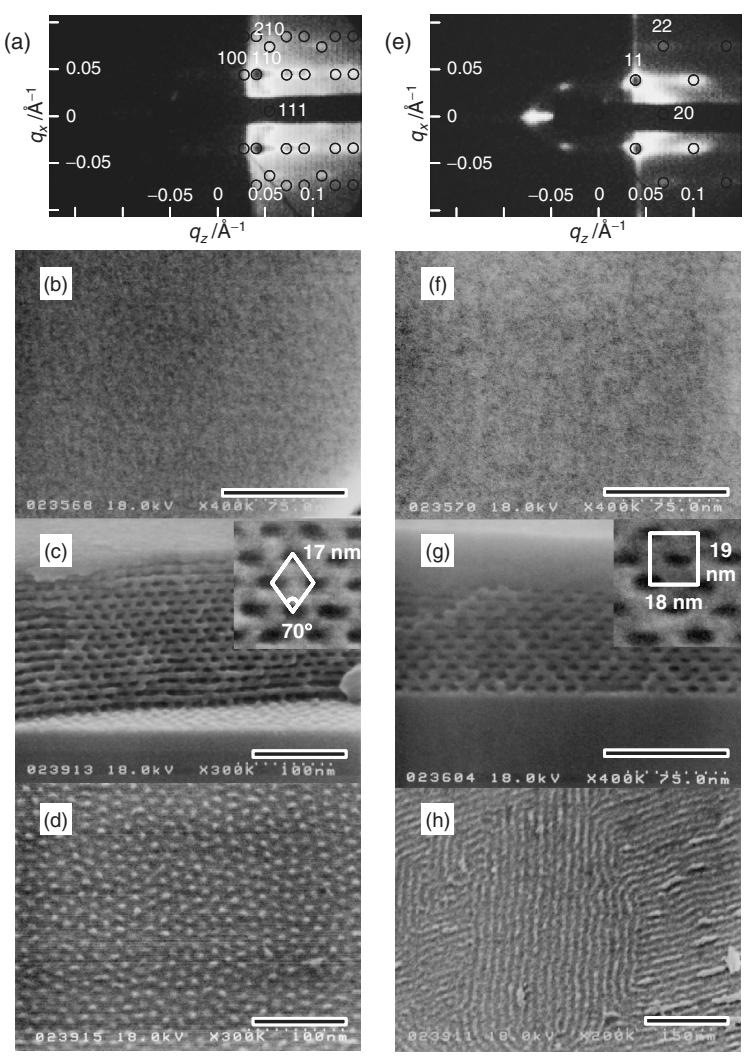

Figure 1. GISAXS patterns (angle of incidence is $0.25^{\circ}$ ) and FESEM images for the (a-d) MSF90(1) and (e-h) MSF45(5). The critical angles for X-ray scattering for the MSF90(1) and MSF45(5) were measured by GISAXS to be $0.18^{\circ}$ and $0.16^{\circ}$, respectively. (b and $f$ ) The top-view images of the film surface, ( $c$ and g) the side-view images of the film, and ( $d$ and $h$ ) the top-view images of the silicon substrate (scale bar; $100 \mathrm{~nm}$ ).

GISAXS and FESEM data for the MSF90(1) shown in Figures 1a-1d are described by rhombohedral $R 3 m$ structure with the (111) oriented perpendicular to the substrate. The overlay of simulated spots is from a NANOCELL simulation with lattice constants $a=16.9 \mathrm{~nm}$ and $\alpha=68^{\circ}$. More recently, Tate and co-workers ${ }^{6}$ and Naik et al. ${ }^{7}$ have reported $R 3 \mathrm{~m}$ structured silica films using $\mathrm{PEO}_{20}-\mathrm{PPO}_{70}-\mathrm{PEO}_{20}$ (Pluronic P123) or CTAB in the presence of 1,3,5-triisopropylbenzene, respectively. To the best of our knowledge, there is no literature on the formation of the $R 3 m$ mesophase using Pluronic F127. Those films have $3 \mathrm{D}$ open pore systems providing high pore accessibility from the surface of the films. Although topologically the MSF90(1) is identical to those reported by Tate and co-workers and Naik et al., the connectivity of the pores in the MSF90(1) is 
different from 3D open system. The periodically arranged silica pillars were observed on the substrate where the film was peeled off (Figure 1d). On the basis of the FESEM observations (data not shown), it was confirmed that the film consists of layered silica parallel to the substrate with pillars through the thickness of the film. This result may be attributed to the formation process of the ordered mesostructure. The lamellar structure is first formed in the early stage of the TEOS infiltration, and then pillars are formed with increasing concentration of TEOS in the film, ${ }^{9 b}$ resulting in no connection of the pores through the thickness of the film.

When the synthetic temperature was $90^{\circ} \mathrm{C}$, ordered structure was obtained after contacting Pluronic F127 films with TEOS for $1 \mathrm{~h}$. No mesophase transition was observed after the formation of $R 3 \mathrm{~m}$ structure. On the contrary, when the synthetic temperature was $45^{\circ} \mathrm{C}$, the synthetic time of $3 \mathrm{~h}$ or more was required until the ordered structure was obtained. Nonporous silica layers exist on the top layer of both the MSF90(1) and MSF45(5) (Figures 1b and 1f). The MSF45(5) is indexed with the (10) oriented rectangular plane group $c 2 \mathrm{~mm}$ (Figures 1e1h). The lattice constants for the MSF45(5) are determined to be $a=19.0 \mathrm{~nm}$ and $b=18.2 \mathrm{~nm}$ which correspond to FESEM image (Figure 1g). The most striking aspect of the results is that the TEOS molecules can be infiltrated into the surfactant film at low temperature such as $45^{\circ} \mathrm{C}$. The channel walls attached on the substrate were observed after the film was peeled off from the substrate (Figure 1h). A domain size of the channel structure seems to be small in the plane parallel to the substrate.

The densities of the MSF90(1) and MSF45(5) were estimated to be 1.27 and $1.13 \mathrm{~g} / \mathrm{cm}^{3}$, respectively, by the average electron density of the films calculated from the critical angle. The mesoporosity of the MSF90(1) and MSF45(5) were found to be 42 and $49 \%$ when the density of the silica framework is assumed to be $2.2 \mathrm{~g} / \mathrm{cm}^{3}$.

The vapor-phase synthesis has two competitive processes; (1) a diffusion (infiltration) and (2) a reaction of TEOS. Diffusion process may result in the increasing $\mathrm{Si}$ /template ratio, whereas the reaction process may produce the rigid silanol network. Infiltration of TEOS may be inhibited by the formation of the rigid silanol network owing to the decreasing of the mobility and flexibility of the silicate framework. The mesophase obtained by the vapor-phase synthesis may be controlled via the silicate/template interfacial curvature adjusting by the quantity of the TEOS infiltration (Figure 2). The thickness of surfactant films is important factor, as well as synthetic temper-

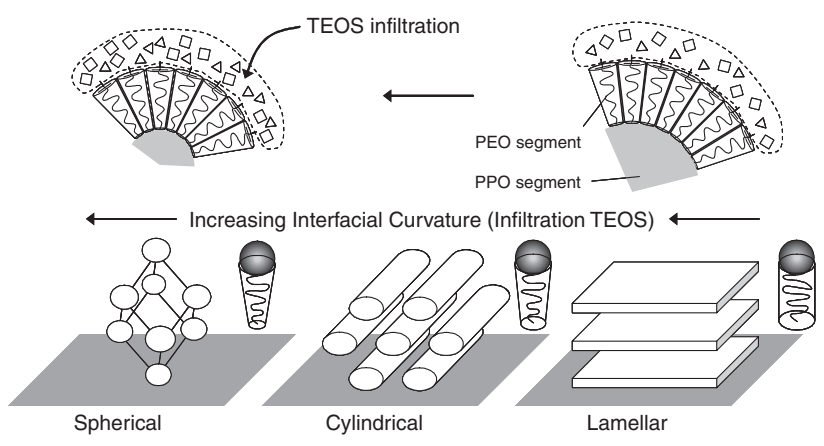

Figure 2. Proposed model for a formation of mesophase via infiltration of TEOS in vapor-phase synthesis. ature and time. The effect of the film thickness for the mesophase topology will be reported in due course.

In conclusion, we have successfully fabricated, by vaporphase synthesis, silica thin films with (10) oriented $c 2 \mathrm{~mm}$ and (111) oriented $R 3 m$ mesophases. We were easily able to control the mesophase via the rate of the TEOS infiltration controlled by the synthetic temperature and time.

The authors wish to acknowledge the National Science Foundation for a Major Research Instrumentation grant (MRI award 0321118-CTS) that was used to establish the Laboratory for In-situ X-ray Scattering from Nanomaterials and Catalysts that was used to collect scattering data. The authors gratefully acknowledge the assistance of the GHAS laboratory and Mr. M. Kawashima at Osaka University with the FE-SEM measurements. S. Tanaka acknowledges the Japan Society for the Promotion of Science (JSPS) Research Fellowships.

\section{References and Notes}

$\dagger$ Present Address: Department of Chemical Engineering, Faculty of Engineering, Kansai University, 3-3-35 Yamate-cho, Suita, Osaka 564-8680.

1 S. Baskaran, J. Liu, K. Domansky, N. Kohler, X. H. Li, C. Coyle, G. E. Fryxell, S. Thevuthasan, R. E. Williford, Adv. Mater. 2000, $12,291$.

2 P. Falcaro, D. Grosso, H. Amenitsch, P. Innocenzi, J. Phys. Chem. B 2004, 108, 10942.

3 A. Gibaud, D. Grosso, B. Smarsly, A. Baptiste, J. F. Bardeau, F. Babonneau, D. A. Doshi, Z. Chen, C. J. Brinker, C. Sanchez, J. Phys. Chem. B 2003, 107, 6114.

4 R. C. Hayward, P. C. A. Alberius, E. J. Kramer, B. F. Chmelka, Langmuir 2004, 20, 5998.

5 P. Innocenzi, L. Malfatti, T. Kidchob, P. Falcaro, S. Costacurta, M. Guglielmi, G. Mattei, V. Belloc, H. Amenitschd, J. Synchrotron Radiat. 2005, 12, 734.

6 a) M. P. Tate, B. W. Eggiman, J. D. Kowalski, H. W. Hillhouse, Langmuir 2005, 21, 10112. b) B. W. Eggiman, M. P. Tate, H. W. Hillhouse, Chem. Mater. 2006, 18, 723.

7 S. P. Naik, T. Yokoi, W. Fan, Y. Sasaki, T.-C. Wei, H. W. Hillhouse, T. Okubo, J. Phys. Chem. B 2006, 110, 9751.

8 C. J. Brinker, Y. F. Lu, A. Sellinger, H. Y. Fan, Adv. Mater. 1999, $11,579$.

9 a) N. Nishiyama, S. Tanaka, Y. Egashira, Y. Oku, K. Ueyama, Chem. Mater. 2003, 15, 1006. b) S. Tanaka, N. Nishiyama, Y. Oku, Y. Egashira, K. Ueyama, J. Am. Chem. Soc. 2004, 126, 4854.

10 M. P. Tate, V. N. Urade, J. D. Kowalski, T.-C. Wei, B. D. Hamilton, B. W. Eggiman, H. W. Hillhouse, J. Phys. Chem. B 2006, 110, 9882.

$110.5 \mathrm{~mL}$ of the template solution (the mole ratios; 0.01Pluronic F127:100ethanol: $100 \mathrm{H}_{2} \mathrm{O}: 0.1 \mathrm{HCl}$ ) was spin-coated on a silicon substrate $\left(2.5 \times 2.5 \mathrm{~cm}^{2}\right.$ piece $)$ at rotating speed of $4000 \mathrm{rpm}$ for $1 \mathrm{~min}$. The resultant film was placed in a closed vessel $(60 \mathrm{~mL})$ along with a separate, small amount of TEOS and $\mathrm{HCl}$, and then the vessel was placed in an oven at 45 or $90^{\circ} \mathrm{C}$. The removal of the template was conducted by calcination at $400^{\circ} \mathrm{C}$ for $5 \mathrm{~h}$ with a heating and cooling ramp of $1{ }^{\circ} \mathrm{C} / \mathrm{min}$. 2D GISAXS measurements were performed using a three-pinhole SAXS camera with a microfocus X-ray source, an Osmic MaxFlux confocal X-ray optic, and a gas-filled 2D multiwire detector at a camera length of $1424 \mathrm{~mm}$. The scattering patterns were calibrated in $q$-space using an isotropic silver behenate powder standard. Because of the presence of an intense specular beam at grazing angles of incidence, aluminum strips were used to attenuate the scattering along the specular plane, blocking the region of $-0.05<q_{z}<0.2 \AA^{-1}$ and $-0.015<q_{x}<0.015 \AA^{-1}$. FESEM measurements were carried out on S-5000L instrument (Hitachi) at an accelerating voltage of $21 \mathrm{kV}$, without any surface coating of the film samples. 